\title{
DÉFICITS SOCIALES EN ADOLESCENTES CON SOBREPESO Y OBESIDAD*
}

\section{SOCIAL DEFICITS IN ADOLESCENTS WITH OVERWEIGHT AND OBESITY}

\author{
Ana Betina Lacunza \\ Universidad del Norte Santo Tomás de Aquino, Argentina \\ Consejo Nacional de Investigaciones Científicas y Técnicas (CONICET), Argentina \\ Silvina Valeria Caballero \\ Universidad del Norte Santo Tomás de Aquino, Argentina \\ Ramiro Salazar \\ Universidad del Norte Santo Tomás de Aquino, Argentina \\ Javier Sal \\ Universidad del Norte Santo Tomás de Aquino, Argentina \\ Josefina Filgueira \\ Universidad del Norte Santo Tomás de Aquino, Argentina
}

\begin{abstract}
Resumen: El objetivo de este trabajo fue identificar las habilidades sociales de adolescentes con sobrepeso y obesidad de contexto urbano y rural de Tucumán. Se realizaron mediciones antropométricas a una muestra de 276 adolescentes de 11 a 20 años, asistentes a escuelas públicas; se les administró además la Batería de Socialización BAS-3 (Silva Moreno y Martorell Pallás, 1989), y una encuesta nutricional como sociodemográfica. Se encontró un $18 \%$ de adolescentes con diagnostico de sobrepeso y obesidad. Este grupo refería mayor retraimiento y ansiedad social al momento de iniciar sus relaciones sociales respecto a sus pares normopeso. Se observó un mayor nivel de ansiedad social y un menor autocontrol en los adolescentes obesos residentes en centros urbanos. Los resultados encontrados destacan la comorbilidad entre estos trastornos de alimentación e indicadores psicopatológicos. Profundizar la investigación de las características de la socialización en adolescentes con Trastornos en la Conducta Alimentaria, particularmente obesidad, permitirá diseñar estrategias de intervención en población urbana y rural.
\end{abstract}

Palabras Clave: adolescentes, sobrepeso, obesidad, retraimiento social, ansiedad social

\begin{abstract}
The objective of this essay was to assess the social skills overweight and obese adolescents who in urban and rural contexts in Tucumán, Argentina. Anthropometric measures were taken from 276 adolescents, 11-20 years old, attending public schools. The Battery of Socialization BAS- 3 (Silva Moreno \& Martorell Pallás, 1989), a nutritional a socio-demographic questionnaires were administered. $18 \%$ of the adolescents were diagnosed with overweight and obesity. This group referred greater shyness and social anxiety when they had to start social relations in comparison to the normal weight counterparts. Obese adolescents residing in urban areas exhibited higher levels of social anxiety and less self-control in obese adolescents who reside in urban areas. The results show the co-morbility between eating disorders and psychopathological indicators. Further research on the characteristics of socialization habits in adolescents with eating disorders, particularly obesity, will allow the design of intervention strategies in urban and rural population.
\end{abstract}

Keywords: adolescents, overweight, obesity, social shyness, social anxiety

* Este estudio ha sido subsidiado por el Consejo de Investigaciones de la Universidad del Norte Santo Tomás de Aquino. Tucumán, Argentina.

\section{Déficits sociales en adolescentes con sobrepeso y obesidad}

En la actualidad los trastornos de la alimentación son un problema sanitario de gran relevancia. Dentro de ellos, el sobrepeso y la obesidad se consideran síndromes complejos mutifactoriales; para algunos autores su etiología está relacionada con la occidentalización de los hábitos alimentarios y el sedentarismo mientras que otros postulan su condicionamiento pre-edipico asociado a la que se ha llamado re-

Correspondencia: Dra. Ana Betina Lacunza. Prof. Asociada a la Cátedra Evaluación Psicológica, Universidad del Norte Santo Tomás de Aquino, Argentina. Correo Electrónico: betinalacunza@conicet.gov.ar 
lación psicosomática (Deckelbaum \& Williams, 2001). Otra variable relevante en la etiología es el contexto cultural, que puede estar mediando entre las variables psicológicas, biológicas y la obesidad. Aguirre (2011) sostiene que distintos procesos sociales (económicos, demográficos, culturales) son los que determinan una particular constelación de factores de riesgo, tales como la abundancia de energía barata, barreras arquitectónicas al movimiento, construcción del gusto, entre otros, que conducen a la epidemia de la obesidad y sus comorbilidades.

Tal es la prevalencia de obesidad a nivel mundial que la Organización de Naciones Unidas (ONU) ha definido que el tema es una cuestión de Estado, puesto que la patología afecta a más personas que la desnutrición. Según Oliver de Schutter, relator de la ONU sobre alimentación, el $65 \%$ de la población mundial vive en países donde la obesidad produce más muertes que las alteraciones nutricionales por déficit. Esta Organización estimó que 1300 millones de personas, es decir el $20 \%$ de la población, sufría sobrepeso en todo el mundo, siendo la causante de alrededor de tres millones de muertes anuales (La Obesidad ya es tan Grave como el Hambre, Clarín, 7 de marzo de 2012). Además, este informe destaca la responsabilidad que tiene la industria alimenticia y la publicidad descontrolada que incita al consumo de alimentos adictivos, como las apreciaciones estéticas de los distintos países occidentales que resaltan a la delgadez como signo de belleza y vinculan el sobrepeso a la fealdad.

Al analizar la prevalencia de sobrepeso y obesidad en América Latina y el Caribe, la Comisión Económica para América Latina (CEPAL, 2009) indicó que Chile, Perú, Venezuela y Argentina, entre 28 países de la región, fueron los que presentaron los peores indicadores de estas alteraciones alimentarias en población infanto-juvenil. Estos datos coinciden con los hallazgos de la Segunda Encuesta Nacional de Factores de Riesgo, realizada en Argentina (Ferrante et al., 2011) y con los aportes de Elgart et al. (2010) quienes señalaban un $49,6 \%$ de jóvenes y adultos con sobrepeso u obesidad. En este estudio se observó que ambas patologías alimentarias registraban aumento, particularmente un $53.4 \%$ de casos con exceso de peso y un aumento de la obesidad en un $18 \%$ en la región Noroeste del país.
Por su parte, el Centro de Estudios sobre Nutrición Infantil (CESNI) y la Sociedad Argentina de Obesidad y Trastornos Alimentarios (SAOTA) realizaron un relevamiento a nivel nacional a fin de elaborar un mapa de la prevalencia de obesidad infanto-juvenil en Argentina. Sobre un total de 119.932 casos analizados en todo el país se encontró que el 31.9\% de preescolares, escolares y adolescentes registraban sobrepeso y obesidad. Respecto a la franja etaria de adolescencia, el $27.3 \%$ de estos presentaba un diagnóstico de sobrepeso y obesidad (CESNI, SAOTA, 2012). Un estudio con adolescentes escolarizados de 14 a 18 años de la ciudad de Lobos (Buenos Aires) mostró que el 15.3\% de las mujeres y el $19 \%$ de los varones presentaban sobrepeso mientras que la obesidad fue de $3.5 \%$ y $1.9 \%$ respectivamente (Mulassi, Borracci, Calderón, Vinay y Mulassi, 2012).

La literatura destaca que ciertas características psicopatológicas tienen un peso relevante en la etiología y mantenimiento de la obesidad, destacando la dimensión multifactorial propia de la patología. Tradicionalmente se ha relacionado la obesidad con la depresión, ansiedad, baja autoestima e insatisfacción corporal (Cebolla, Baños, Botella, Lurbe y Torró, 2011). Sin embargo, estos mismos autores destacan que la evidencia empírica es contradictoria respecto a si, por ejemplo, los niños y adolescentes con depresión tienen mayor probabilidad de desarrollar depresión en la vida adulta (Goodman \& Whitaker, 2002).

A estos indicadores psicopatológicos podría sumarse una dificultad en las relaciones interpersonales, descripta ya por Behar, Rodrigo y Dunny (2006) sobre la menor asertividad que mostraban adolescentes y jóvenes mujeres con Trastornos en la Conducta Alimentaria (TCA) que sus pares de población general. Un estudio realizado con adolescentes de 12 a 16 años mostró diferencias significativas en la autopercepción negativa de la imagen corporal, la obsesión por la delgadez y la mayor vulnerabilidad percibida en los adolescentes con obesidad (Hernández Martínez, 2010). Esta mayor vulnerabilidad puede generar dificultades en el contacto social con pares y adultos. Al respecto, Strauss y Pollack (2003) marcan que esto generaría que los adolescentes con exceso de peso fueran víctimas de maltrato por parte de sus compañeros y familiares, exclusión social, 
prejuicio y discriminación. Estas afirmaciones son congruentes con la propuesta de MacElroy, Kotwall, Malhotra, Nelson, Keck y Nemeroff (2004) quienes postulan una mayor prevalencia de baja autoestima y depresión en jóvenes con sobrepeso y obesidad.

En el caso de adultos, un estudio realizado con mujeres brasileñas obesas en tratamiento médico indicó que el grupo clínico mostraba mayores dificultades en las habilidades sociales asertivas relacionadas con la alimentación, por ejemplo, en el autocontrol, lo que dificulta la superación de la enfermedad. Asimismo las puntuaciones en las habilidades sociales generales eran similares al grupo control, sobre todo para el desenvolvimiento social con conocidos (Correia, Prette del y Prette del, 2004). Estos resultados apoyan las nociones y creencias de que las gorditas son simpáticas y agradables, particularmente con su círculo social más próximo (Miller, Rothblum, Brand \& Felicio, 1995).

A nivel local, estudios previos han encontrado asociaciones entre indicadores de riesgo alimentario (comportamiento de tipo bulímico e insatisfacción corporal) y dimensiones inhibidoras de la socialización (retraimiento y ansiedad social /timidez) en adolescentes urbanos de nivel socioeconómico bajo (NES) (Lacunza et al., 2010). La presencia de comportamientos de retraimiento social en los adolescentes de NES bajo de Tucumán también fueron descriptos por Contini, Cohen, Coronel y Mejail (2012), quienes encontraron que el $19.8 \%$ de su muestra refería sentimientos de vergüenza, timidez y ansiedad social, comportamientos incluidos en los llamados problemas internalizantes de la psicopatología taxonómica de Achenbach (1966). En un relevamiento de adolescentes de zonas rurales de Tucumán se encontró que el $13 \%$ presentaba sobrepeso y la obesidad, en este estudio también se observaron conductas alimentarias de riesgo como temor a engordar e insatisfacción corporal (24\%) en los adolescentes y éstas se presentaron independientes de la malnutrición (Lacunza, Caballero, Filgueira, Sal y Salazar, 2012).

El objetivo del presente trabajo fue identificar las habilidades sociales de adolescentes con sobrepeso y obesidad de contexto urbano y rural de Tucumán. Se partió de la hipótesis que los adolescentes del grupo clínico mostrarían mayores dificultades en sus habilidades respecto al grupo control.

\section{Método}

\subsection{Participantes}

375 adolescentes, entre 11 y 20 años, de contexto urbano (San Miguel de Tucumán) y rural (Burruyacu y Gastona Sud) de la provincia de Tucumán (Argentina). Todos los adolescentes pertenecían a instituciones educativas públicas, ubicadas en zonas de nivel socioeconómico bajo. El 70\% pertenecía a zonas rurales. La edad promedio fue de 14 años (DE: 1.63). El $58 \%$ fueron mujeres. El grupo clínico estuvo conformado por adolescentes con diagnóstico antropométrico de sobrepeso (percentilo superior a 85) y obesidad (percentilo superior a 95) según parámetros de la Center of Desease Control and Prevention (CDC, 2002). El grupo control incluyó a todos los adolescentes con diagnóstico de normopeso según los mismos estándares.

\subsection{Instrumentos}

- Batería de Socialización BAS-3 (Silva Moreno y Martorell Pallás, 1989): está integrada por 75 ítems, aplicable a adolescentes de 11 a 19 años de edad, de autoadministración individual con dos opciones de respuesta (Si-No). Permite obtener un perfil del comportamiento social a partir de sus cinco escalas de socialización: Consideración con los demás (Co), Autocontrol en las Relaciones Sociales (Ac), Retraimiento Social (Re), Ansiedad Social/Timidez (At) y Liderazgo (Li). A éstas se le suma una escala de Sinceridad (S), que permite analizar la consistencia interna de las respuestas a las escalas. Los estudios de fiabilidad mostraron una consistencia interna satisfactoria (coeficientes alpha de .73 a .82 para las distintas escalas). El test-retest, en un intervalo de cuatro meses, confirmó una estabilidad temporal adecuada para Autocontrol $(r=.66)$ y Liderazgo $(r=.61)$, siendo menor para Consideración con los demás $(r=.42)$ y Retraimiento $(r=.43)$ (Silva Moreno y Martorell Pallás, 1989). Un estudio local analizó las propiedades psicométricas de la BAS-3 en población adolescente de S.M. de Tucumán, encontrándose que al análisis factorial exploratorio identificaba cinco factores (sin incluir la escala S) que explicaban el $26.65 \%$ de la varianza total. Además un análisis desde la propuesta del modelo jerárquico de la socialización propuesta 
por los autores mostraba una solución de dos factores que explicaban el $53.13 \%$ de la varianza. Las asociaciones estadísticas observadas daban cuenta de la presencia de factores primarios, secundarios y terciarios de la socialización. El análisis de fiabilidad indicó una consistencia interna satisfactoria en las escalas facilitadoras e inhibidoras (coeficiente alpha de Cronbach: Consideración .688, Autocontrol .676, Retraimiento .685, Ansiedad-timidez .721, Liderazgo .520 y Sinceridad .608) (Lacunza, Caballero y Contini, 2012).

- Valoración del estado nutricional a partir indicadores antropométricos: talla $(T)$, peso $(\mathrm{P})$, para la determinación del Índice de Masa Corporal (IMC) considerando los estándares de valoración para sexo y edad propuestos por la CDC (2002).

- Encuesta sociodemográfica y nutricional: el apartado sociodemográfico incluyó aspectos vinculados al grupo familiar del adolescente (tipo de grupo familiar, cantidad de hermanos, posición entre los hermanos) y el nivel socioeconómico (nivel educativo y ocupación de los padres). El apartado nutricional incluyó preguntas referidas a percepción corporal, realización de dietas, preferencias alimentarias, consumo de alimentos protectores y un recordatorio de 24 horas sobre consumo de alimentos.

\subsection{Procedimiento}

Se llevó a cabo una investigación descriptiva de diseño transversal con muestreo no probabilístico, intencional (Hernández Sampieri, Fernández Collado y Baptista Lucio, 2010). Se realizaron entrevistas con directivos de las escuelas para acordar pautas de trabajo. Se administró la BAS-3 de forma grupal, previa autorización de los padres (consentimiento informado). La valoración antropométrica se realizó de modo individual en el establecimiento educativo. Ambas mediciones se realizaron entre junio y noviembre de 2010. Acompañaron a los profesionales del equipo de investigación alumnos avanzados de la Lic. en Nutrición y Psicología para la administración de dichas pruebas y mediciones. Posteriormente se analizó descriptivamente la BAS-3 y las mediciones antropométricas. Se calculó el índice de masa corporal (IMC) con la fórmula: IMC $=$ peso $(\mathrm{kg}) /$ estatura $^{2}(\mathrm{~m})$. Como criterios de clasificación y estándar de referencia se utilizaron las correspondientes a las tablas de la CDC (2002), se calculó el z-score y el percentilo de cada adolescente utilizando como parámetros: mayor al percentilo 85 se consideró sobrepeso y mayor al percentilo 95 obesidad. Las puntuaciones obtenidas fueron analizadas con el paquete estadístico PASW-18. Para esta presentación no se han considerado datos de la encuesta nutricional y sociodemográfica.

\section{Resultados}

En un primer momento se analizaron las mediciones antropométricas. Para esta instancia sólo participaron 276 adolescentes debido a su negativa para pesarse y medirse (a pesar de contar con el consentimiento informado de sus padres), es decir, el $22 \%$ de la muestra total no participó de esta evaluación. Se encontró que el $22.82 \%$ de los adolescentes presentaba malnutrición por exceso y/o déficit nutricional (Tabla 1). Cabe destacar que 49 adolescentes conformaron el grupo clínico de sobrepeso y obesidad, siendo mayor el número de mujeres con patologías (mujeres $=26$, varones $=23$ ) .

Tabla 1

Categorías del estado nutricional

\begin{tabular}{lcc}
\hline Categorías IMC & F & $\%$ \\
\hline Normonutrido & 213 & 77.17 \\
Desnutrido & 4 & 1.44 \\
Sobrepeso & 24 & 8.69 \\
Obesidad & 35 & 12.68 \\
\hline
\end{tabular}

Posteriormente se analizaron las habilidades sociales de la muestra completa según el estado nutricional. Cabe destacar que sólo 262 adolescentes con mediciones antropométricas accedieron a responder la BAS-3. El análisis de Anova mostró la existencia de diferencias estadísticas significativas en la escala $\operatorname{Re}[F(3,258)=2.24, p=.028]$, por lo que los adolescentes con obesidad referían ser más retraídos al momento de iniciar relaciones sociales (Tabla 2). Cabe destacar que solo cuatro adolescentes fueron diagnosticados con déficit nutricional (desnutrición).

Teniendo en cuenta la zona de residencia, se compararon las habilidades sociales de los adolescentes normonutridos con aquellos con sobrepeso y obesidad. Se encontró que los 
adolescentes rurales con sobrepeso y obesidad referían ser más retraídos que sus pares sin exceso nutricional (Tabla 3). En tanto, los análisis univariados mostraron que los adolescentes urbanos con obesidad mostraban mayor retraimiento y ansiedad social/timidez y menor autocontrol. Sólo se encontraron diferencias estadísticas en la dimensión Ac, a favor del grupo de adolescentes con sobrepeso $(F=2.66$, $g l=3, p=.05)$ (Tabla 4).

Tabla 2

Escalas de socialización BAS-3 según estado nutricional. Análisis univariado, muestra completa

\begin{tabular}{|c|c|c|c|c|c|c|c|c|c|}
\hline \multirow{3}{*}{$\begin{array}{c}\text { Dimensiones } \\
\text { BAS-3 }\end{array}$} & \multicolumn{8}{|c|}{ Estado Nutricional } & \multirow[b]{3}{*}{$F$} \\
\hline & \multicolumn{2}{|c|}{$\begin{array}{c}\text { Normonutrido } \\
\text { (n: 209) }\end{array}$} & \multicolumn{2}{|c|}{$\begin{array}{l}\text { Desnutrido } \\
\text { (n: 4) }\end{array}$} & \multicolumn{2}{|c|}{$\begin{array}{c}\text { Sobrepeso } \\
\text { (n: 24) }\end{array}$} & \multicolumn{2}{|c|}{$\begin{array}{c}\text { Obesidad } \\
\text { (n: 25) }\end{array}$} & \\
\hline & M & $D E$ & M & $D E$ & M & $D E$ & M & $D E$ & \\
\hline Co & 11.3 & 2.38 & 10 & 4.96 & 11.46 & 2.39 & 10.36 & 1.28 & .478 (ns) \\
\hline Ac & 9.19 & 2.68 & 7.33 & 1.52 & 10.11 & 1.61 & 9.64 & 1.96 & .983 (ns) \\
\hline Li & 7.12 & 2.52 & 4.33 & 3.21 & 6 & 1.73 & 6.09 & 2.8 & $2.15(\mathrm{~ns})$ \\
\hline $\mathrm{Re}$ & 4.57 & 3.16 & 2.5 & .70 & 5.11 & 3.75 & 7 & 4.17 & 2.24 (sig) \\
\hline At & 5.58 & 2.63 & 6.33 & 2.51 & 5.8 & 3.18 & 4.91 & 3.04 & .345 (ns) \\
\hline
\end{tabular}

Nota: Co: Consideración con los demás, Ac: Autocontrol, Li: Liderazgo, Re: Retraimiento, At: Ansiedad social/ Timidez.

Nota: $\mathrm{p}<.05$

Tabla 3

Escalas BAS-3 según estado nutricional. Análisis univariado, zona rural

\begin{tabular}{|c|c|c|c|c|c|c|c|}
\hline \multirow{3}{*}{$\begin{array}{c}\text { Dimensiones } \\
\text { BAS-3 }\end{array}$} & \multicolumn{6}{|c|}{ Estado Nutricional } & \multirow[b]{3}{*}{$F$} \\
\hline & \multicolumn{2}{|c|}{$\begin{array}{c}\text { Normonutrido } \\
\text { (n: 143) }\end{array}$} & \multicolumn{2}{|c|}{$\begin{array}{c}\text { Sobrepeso } \\
\text { (n: } 9)\end{array}$} & \multicolumn{2}{|c|}{$\begin{array}{c}\text { Obesidad } \\
\text { (n: 11) }\end{array}$} & \\
\hline & $M$ & $D E$ & $M$ & $D E$ & $M$ & $D E$ & \\
\hline Co & 11.45 & 2.24 & 11.78 & 1.78 & 11.36 & 1.28 & .478 (ns) \\
\hline$A c$ & 9.19 & 2.68 & 10.11 & 1.61 & 9.64 & 1.96 & $.983(\mathrm{~ns})$ \\
\hline $\mathrm{Li}$ & 7.12 & 2.52 & 6 & 1.73 & 6.09 & 2.8 & $2.15(\mathrm{~ns})$ \\
\hline $\mathrm{Re}$ & 4.57 & 3.16 & 5.11 & 3.75 & 7 & 4.17 & 2.24 (sig) \\
\hline At & 5.58 & 2.63 & 5.8 & 3.18 & 4.91 & 3.04 & .345 (ns) \\
\hline
\end{tabular}

Nota: Co: Consideración con los demás, Ac: Autocontrol, Li: Liderazgo, Re: Retraimiento, At: Ansiedad social/Timidez.

Nota: $\mathrm{p}<.05$

Tabla 4

Escalas BAS-3 según estado nutricional. Análisis univariado, zona urbana

\begin{tabular}{cccccccc}
\hline \multirow{2}{*}{$\begin{array}{c}\text { Dimensiones } \\
\text { BAS-3 }\end{array}$} & \multicolumn{2}{c}{$\begin{array}{c}\text { Estado Nutricional } \\
(n: 66)\end{array}$} & \multicolumn{2}{c}{$\begin{array}{c}\text { Sobrepeso } \\
(n: 15)\end{array}$} & \multicolumn{2}{c}{$\begin{array}{c}\text { Obesidad } \\
(n: 14)\end{array}$} \\
\cline { 2 - 8 } & $M$ & $D E$ & $M$ & $D E$ & $M$ & $D E$ & $F$ \\
\cline { 2 - 8 } Co & 10.95 & 2.65 & 11.26 & 2.73 & 9.64 & 3.97 & $.960(\mathrm{~ns})$ \\
Ac & 9.79 & 2.3 & 10.35 & 2.67 & 8.21 & 2.91 & $2.66(\mathrm{sig})$ \\
$\operatorname{Re}$ & 3.73 & 2.01 & 2.86 & 2.16 & 4.14 & 2.38 & $1.51(\mathrm{~ns})$ \\
$\mathrm{At}$ & 5.67 & 2.69 & 6.6 & 3.86 & 6.28 & 2.39 & $1.13(\mathrm{~ns})$ \\
$\mathrm{Li}$ & 7.48 & 2.28 & 7 & 2.2 & 6.28 & 2.7 & $1.35(\mathrm{~ns})$ \\
\hline
\end{tabular}

Nota: Co: Consideración con los demás, Ac: Autocontrol, Li: Liderazgo, Re: Retraimiento, At: Ansiedad social/ Timidez.

Nota: $p<.05$ 


\section{Discusión}

El objetivo del presente trabajo fue identificar las habilidades sociales de adolescentes con sobrepeso y obesidad de contexto urbano y rural de Tucumán. Las mediciones antropométricas mostraron que el $23 \%$ de los adolescentes participantes presentaba malnutrición ( $9 \%$ sobrepeso, $13 \%$ obesidad y $1 \%$ desnutrición). Estos resultados se relacionan con la evidencia empírica del mapa sobre obesidad en Argentina en población adolescente (sobrepeso 17.4\%, obesidad 9.9\%) descripto por CESNI y SAOTA (2012) como los datos de la última Encuesta Nacional de Factores de Riesgo (Ferrante et al., 2011) y las observaciones descriptas, a nivel mundial, por el área de Nutrición de la ONU. El aumento de déficits nutricionales, particularmente por exceso, da cuenta de los cambios en los patrones alimentarios y en los estilos de vida que han generado conductas obesogénicas en la población, particularmente en niños y adolescentes. Según autores locales, estos comportamientos se caracterizan por una disminución de alimentación natural, mayor consumo de grasas, hidratos de carbono refinados, gaseosas, jugos artificiales y comidas rápidas (Pérez, Sandoval, Schneider y Azula, 2008; Torresani et al., 2006). A esto se suma el sedentarismo, la práctica de juegos pasivos y el uso indiscriminado de productos tecnológicos.

El sobrepeso y la obesidad en niños y adolescentes es un problema importante para la salud pública de países industrializados y subdesarrollados, particularmente la obesidad en contextos de pobreza, tal como se describen en los resultados del presente estudio. Se ha encontrado que la obesidad en sujetos con carencias socioeconómicas se asocia a episodios de desnutrición en edades tempranas, incluso en la vida intrauterina. Estas carencias generan respuestas de adaptación a los escasos aportes de energía que en etapas sucesivas de mayores ingestas pueden producir un aumento de peso. Sumado a ello, la accesibilidad, casi exclusiva, a alimentos ricos en azúcares y grasas (de menor costo que aquellos de nutrientes esenciales) y el consumo de comidas rápidas definen los patrones de consumo y pautas de comensalidad en este sector de la población (Pedraza, 2009).

Si bien el diagnóstico estuvo dado por mediciones antropométricas, varios alumnos no participaron en esta instancia, dato que revelaría una cierta inconformidad con el propio cuerpo como un posible malestar e incomodidad respecto a ser observado por otro. Esta insatisfacción corporal, propia de la transición de la etapa adolescente, puede adquirir matices psicopatológicos si se acompaña de otras conductas alimentarias de riesgo y de percepciones negativas respecto a si mismo. Un respaldo de estos comportamientos adolescentes es el de la cultura, particularmente la occidental, en la que se exalta, por un lado, un ideal de delgadez extrema y, por otro lado, se muestra constantemente un medio obesogénico. Este ideal de belleza y delgadez pareciera instituirse como modelo de influencia universal, puesto que resultados preliminares locales indicaron la presencia de una insatisfacción corporal tanto en adolescentes urbanos como rurales (Lacunza et al., 2010; Lacunza, Caballero y Contini, 2011).

Se ha descripto que la obesidad es una patología multidimensional en el que sus efectos a nivel psicosocial se vinculan a baja autoestima, escaso control de impulsos, temor a no ser aceptado, inadaptación social, bloqueos afectivos, pasividad, sumisión, inseguridad, sentimientos de culpa, entre otros. La obesidad puede ser utilizada como una protección ante un contexto amenazante y frustrante, lo que limitaría la práctica social y el desarrollo de habilidades sociales. De allí, el interés de este estudio de describir la autopercepción del adolescente obeso y con sobrepeso respecto a sus comportamientos sociales. El análisis de los resultados permitió observar la tendencia de los adolescentes obesos y con sobrepeso a mostrar habilidades sociales del estilo inhibido. En este estudio, la escala Retraimiento social del BAS-3 registró puntajes más altos para estos grupos, lo que mostraría sus déficits para establecer relaciones socialmente satisfactorias con pares y adultos. Sus comportamientos se caracterizarían por una gran tendencia a evitar los contactos sociales con otras personas en diversas situaciones sociales. Estos hallazgos son coincidentes con los resultados de Galarsi, Ledesma, Bortoli De y Correche (2009) para población adolescente escolarizada de San Luis (Argentina). Para estos autores los rasgos de Introversión aumentan en las mujeres obsesionadas por la delgadez. Además, los indicadores empíricos coinciden con los aportes de Békei (1984) al señalar como rasgos característicos del sujeto obeso el retraimiento, el desvalimiento, la escasa tolerancia a la frustración y la pasividad. 
La influencia del contexto es fundamental al momento de analizar las habilidades sociales, puesto que lo socialmente válido en una situación puede no serlo en otra. Este estudio encontró un perfil social según la distinción urbano-rural; los adolescentes rurales con obesidad mostraban habilidades sociales del estilo inhibido, particularmente los puntajes altos en la escala Retraimiento social de la BAS-3. Por su parte, los adolescentes urbanos referían un menor autocontrol, que se manifestarían en un pobre control de sus impulsos y tendencias autoagresivas. Esto ya fue planteado por Bekéi (1984) al afirmar que las personas obesas no discriminan sensaciones corporales (como hambre-apetito) lo que se manifiesta en el comer compulsivamente. Paradójicamente este comportamiento compulsivo les aporta seguridad ante aquellas situaciones que reactiven sus inseguridades.

Respecto a la mediación de aspectos contextuales, cabe analizar con mayor profundidad si este estilo inhibido de habilidades sociales es característico en adolescentes con sobrepeso y obesidad de contextos de pobreza o si también está presente en adolescentes con obesidad de otros niveles socioeconómicos. Esto constituye una limitación del estudio puesto que se trabajó solo con población adolescente asistente a establecimientos educativos de nivel socioeconómico bajo.

Los resultados encontrados en esta investigación son coincidentes con tendencias nacionales e internacionales que marcan comorbilidad entre trastornos de alimentación, particularmente sobrepeso y obesidad, y alteraciones en distintas áreas de la personalidad o patologías mentales. Los datos descriptos destacan los déficits en las habilidades sociales de los adolescentes, urbanos y rurales, con sobrepeso y obesidad. Su pertenencia a niveles socioeconómicos más desfavorecidos incluye a estos adolescentes en el llamado grupo de gordos pobres (Aguirre, 2004). Sin embargo, no puede atribuirse que sólo la variable socioeconómica sea el principal determinante de la patología, sin considerar otros factores sociales. Tal como plantea Aguirre (2011) tomando los aporte de Nestlé (2006), no podrá abordarse la epidemia de la obesidad mientras se la considere un problema individual y no de la sociedad que "les sirve en bandeja las condiciones de su obesidad" (p. 59).
De allí la importancia de profundizar la investigación de las variables mediadores de la obesidad en población adolescente, particularmente las características de la socialización. Además, la detección de estos indicadores de riesgo impedirá su cristalización en patologías psicológicas que exacerben el estado nutricional del adolescente.

\section{Referencias}

Achenbach, T. (1966). The classification of children's psychiatric syntoms: A factor-analitic study. Psychological Monographs, 80, 1-37.

Aguirre, P. (2004). Ricos flacos y gordos pobres. La alimentación en crisis. Buenos Aires: Capital Intelectual.

Aguirre, P. (2011). La construcción social del gusto en el comensal moderno. En M. Katz (Ed.), Comer. Puentes entre la alimentación y la cultura. Buenos Aires: Libros del Zorzal.

Behar, R., Rodrigo, M. G., y Dunny C. Z. (2006). Trastorno de la conducta alimentaria y asertividad. Revista Médica de Chile, 134, 312-319.

Békei, M. (1984). Trastornos psicosomáticos en la niñez y adolescencia. Buenos Aires: Nueva Visión.

Cebolla, A., Baños, R., Botella, C., Lurbe, E., y Torró, M. (2011). Perfil psicopatológico de niños con sobrepeso u obesidad en tratamiento de pérdida de peso. Revista de Psicopatología y Psicología Clínica, 16(2), 125-134.

Centro de Estudios sobre Nutrición Infantil, Sociedad Argentina de Obesidad y Trastornos Alimentarios (Octubre, 2012). Ira Jornada de Obesidad Infantil. Hacia el mapa de la obesidad en Argentina. Recuperado de http://www.cesni.org.ar/Content/pres.mapa12.pdf

Comisión Económica para América Latina y el Caribe (Noviembre, 2009). Crisis alimentaria y su impacto en América Latina. Trabajo presentado en Segundo Seminario Internacional de Alimentación Escolar. Recuperado de http://www.larae.org/home/contenido/ congresos_y_seminarios/II\%20Seminario/doc/Informe $\% 20 y \% 20$ avances $\% 20$ de $\% 20$ acuerdos $\% 20$ II\%20Seminario.pdf

Center of Desease Control and Prevention (2002). 2000 CDC growth charts for the United States: Methods and development. Vital Health Statistics 11(246). Recuperado de http://www.cdc.gov/ growthcharts/2000growthchart-us.pdf

Contini, N., Cohen I. S., Coronel, P. y Mejail, S. (2012). Agresividad y retraimiento en adolescentes. Ciencias Psicológicas, VI(1), 17-28.

Correia, S., Prette del, Z., y Prette del, A. (2004). Habilidades sociais en mulheres obesas: um estudo exploratorio. PSICO-USF, 9(2), 201-210.

Deckelbaum, R. J., \& Williams, C. L. (2001). Childhood obesity: the health issue. Obesity Research, 9, 239S-243S.

Elgart, J., Pfirter, G., González, L., Caporale, J., Cormillot, A., Chiappe, M. L., y Gagliardino, J. (2010). Obesidad en Argentina: Epidemiología, morbimortalidad e impacto económico. Revista Argentina de Salud Pública, 1(5), 6-12. 
Ferrante, D., Linetzky, B., Konfino, J., King, A., Virgolini, M., y Laspiur, S. (2011). Encuesta Nacional de Factores de Riesgo 2009: Evolución de la Epidemia de Enfermedades Crónicas No Transmisibles en Argentina. Revista Argentina de Salud Pública, 2(6), 34-41.

Galarsi, M. F., Ledesma, C., Bortoli De, M. A., y Correche, M. S. (2009). Rasgos de Personalidad y Trastornos de la Conducta Alimentaria en Estudiantes Universitarias. Fundamentos en Humanidades, 1(19), 157-166.

Goodman, E., \& Whitaker, R. (2002). A prospective study of the role of depression in the development and persistence of adolescent obesity. Pediatrics, 110(3), 497-504.

Hernández M. H. (2010). Modelo de creencias de salud y obesidad. Un estudio de los adolescentes de la provincia de Guadalajara. (Tesis Doctoral) Universidad de Alcalá, Madrid. Recuperado de http://hdl.handle. net/10017/9024

Hernández Sampieri, R., Fernández Collado, C., y Baptista Lucio, P. (2010). Metodología de la Investigación (5 ${ }^{\mathrm{a}}$ ed.). México: Mc Graw Hill.

La Obesidad ya es tan Grave como el Hambre (20 de enero de 2012).Diario Clarín.Recuperado de http://www.clarin.com/salud/obesidad-grave-hambre_0_659334225.html

Lacunza, A., Caballero, V. \& Contini, N. (2012). Adaptación y evaluación de las propiedades psicométricas de la BAS-3 para población adolescente de Tucumán, Argentina. Manuscrito presentado para su publicación.

Lacunza, A., Caballero, V., y Contini, N. (Setiembre, 2011). Insatisfacción corporal y déficits en las habilidades sociales de adolescentes rurales. Memorias del Il Congreso de Psicología del Tucumán. S.M. de Tucumán.

Lacunza, A., Caballero, V., Cordero, L., Sal, J., Salazar B. R., y Filgueira, J. (2010). Identificación temprana de factores de riesgo desde un abordaje interdisciplinario: conductas alimentarias de riesgo y déficits de socialización en adolescentes tucumanos. Psicodiagnosticar, Revista de la Asociación Argentina de Estudio e Investigación en Psicodiagnóstico, 20, 99-112.
Lacunza, A., Caballero, V., Filgueira, J., Sal, J. y Salazar Burgos, R. (2012). Riesgo de trastornos alimentarios en adolescentes de contexto rural. Insatisfacción corporal y déficits en habilidades sociales. Revista Acta Psiquiátrica y Psicológica de América Latina, 58 (3), 157-163.

McElroy, S. L., Kotwal, R., Malhotra, S., Nelson, E. B., Keck, P. E. \& Nemeroff, C. B. (2004). Are mood disorders and obesity related? A review for the mental health professional. Journal Clinical Psychiatry, 65, 634-651

Miller, C. T., Rothblum, E. D., Brand, P. A. \& Felicio, D. M. (1995). Do obese women have poorer social relationships than non-obese women? Reports by self, friends, and co-workers. Journal of Personality, $63,65-85$.

Mulassi, A., Borracci, R., Calderón, J., Vinay, P. y Mulassi, M. (2012). Redes sociales de tabaquismo, consumo de alcohol y obesidad en adolescentes escolarizados de la ciudad de Lobos. Archivos Argentinos de Pediatría, 110(6), 474-482.

Nestlé, M. (2006). Food marketing and childhood obesity. A matter of policy. New England Journal Medicine, 24, 2527-2529.

Pedraza F. D. (2009). Obesidad y pobreza: marco conceptual para su análisis en Latinoamérica. Saúde e Sociedade, 18(1), 103-117.

Pérez, E. C., Sandoval, M. J., Schneider, S. E. y Azula, L. A. (2008). Epidemiología del sobrepeso y la obesidad en niños y adolescentes. Revista de Posgrado de la Vía Cátedra de Medicina, 179, 16-20.

Silva Moreno, F. y Martorell Pallás, M. C. (1989). BAS-3 Batería de Socialización (Autoevaluación). Madrid: TEA Ediciones.

Strauss, R. \& Pollack, H. (2003). Social marginalization of overweight children. Archivos de Pediatría Adolescente, 157, 746-752

Torresani, M., Raspini, M., Acosta Sero, M., Giusti, L., García, C., Español, S...Rodríguez, L. (2006). Prevalencia de conductas obesogénicas en escolares y adolescentes de Capital y Gran Bs. As. Actualización en Nutrición, 7(4), 53- 62.

Para citar este artículo:

Lacunza, A. B., Caballero, S. V., Salazar, R., Sal, J., y Filgueira, J. (2013). Déficit sociales en adolescentes con sobrepeso y obesidad. Ciencias Psicológicas VII (1): 25 - 32. 